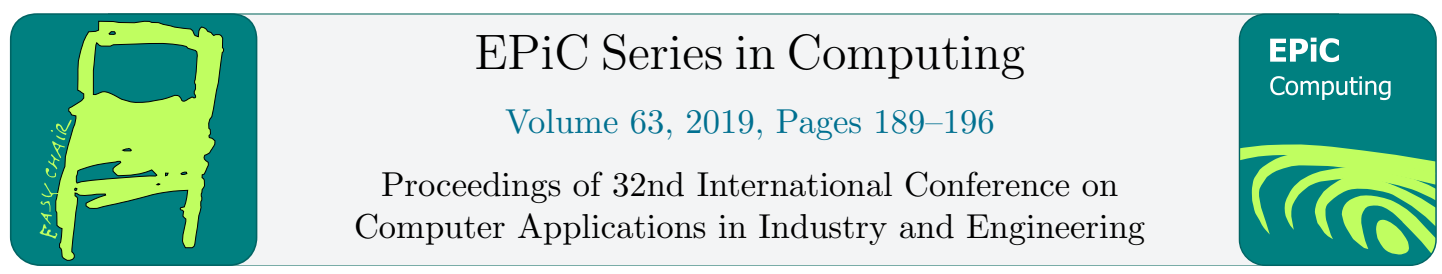

\title{
Augmented Reality Application to Enhance Training of Lumbar Puncture Procedure: Preliminary Results
}

\author{
Nikolay Sargsyan ${ }^{1}$, Robertson Bassy ${ }^{1}$, Xin Wei $^{1}$, Sathish Akula ${ }^{1}$, Jueting Liu ${ }^{1}$, \\ Cheryl Seals ${ }^{1}$, and Jeremy White ${ }^{2}$ \\ 1 Auburn University, Auburn, Alabama, U.S.A. \\ nzs0067@auburn.edu, rzb0053@auburn.edu, xzw0059@auburn.edu, sza0096@auburn.edu, \\ jzl0122@auburn.edu, sealscd@auburn.edu \\ 2 Edward Via College of Osteopathic Medicine - Auburn Campus, Auburn, Alabama, U.S.A. \\ jjwhite@auburn.vcom.edu
}

\begin{abstract}
With the recent rapid development of Augmented Reality (AR) headsets, new possibilities emerge for applications of AR technologies. Today, publicly available AR headsets provide novel storytelling platforms, expand the vision of doctors and engineers, remove boundaries of educational processes and assist humanity in multiple endeavors.

Our research encompasses various levels of the learning process by examining design principals and developing reliable software for multiple educational applications. This paper focuses on the software development process for an AR program that teaches the medical procedure known as the Lumbar Puncture. Our team utilized the Meta 2 headset by Metavision to create a software application to enhance the student experience and extend training effectiveness. We will discuss the requirements and specification for the future application, describe the development process and issues encountered for the current version of the application, as well as present preliminary results of testing and evaluation.
\end{abstract}

\section{Introduction}

Augmented Reality allows for virtual presences to be blended into the user's reality with minimal interference compared with virtual reality (VR), which creates a totally virtual environment for users [9]. Augmented Reality has become a complex system, which shows a virtual presence to users by using targets as markers, geolocation, object recognition and/or voice activation [9]. Today, after more then roughly 60 years of evolution, Augmented Reality is used in various fields like medicine, military, tourism and marketing, where it assists with many different tasks such as data visualization, environment simulation and many others [4], [14].

Medical professionals traditionally are trained in multitudes of operations and minor procedures to provide proper services for patients. For ages, different methods to simulate actual human body and medical operation environments were used, with contemporary market providing a broad choice of products with different levels of detail, animation and provided feedback [13], [11]. With the new AR headsets and supporting tools being introduced into the market,

Q. Yuan, Y. Shi, L. Miller, G. Lee, G. Hu and T. Goto (eds.), CAINE 2019 (EPiC Series in Computing, vol. 63), pp. 189-196 
and technology becoming more precise and cheaper, we look forward to growth in actual usage of AR headsets for purposes of medical training and education with numerous software applications designed for AR headsets already being subjects of research [15], [1], [5], [8].

The Lumbar Puncture procedure, also known as spinal tap, is a medical procedure, during which a thin hollow needle is inserted into the space surrounding the spinal column in the lower back of the patient to collect cerebrospinal fluid (CSF). The importance of this operation is that the analysis of CSF can be used for diagnosing various serious infections and disorders of the central nervous system, such as meningitis, encephalitis and neurological diseases. Besides, doctors can directly insert medicine into the spinal cord for some special treatments by the Lumbar Puncture, such as spinal anesthesia before a surgery. The operation must be executed carefully and safely as it may cause both benign and serious side effects if done incorrectly.

The current process of the training for the Lumbar Puncture procedure include as checklist of actions that the student must perform under the supervision of a training specialist. The checklist requires student to:

1. orally state the the medical uses, contraindications and adverse effects of the procedure

2. orally state the steps needed to perform the procedure

3. perform the procedure with real medical tools and a specially designed manikin that simulates the lower back of the human body

The goal of our research is to build an AR system that will serve as a library for the student, augment the existing training tools for a more realistic experience, and test and provide feedback on student actions. This system will not only make the training environment closer to a real one, but also become a visual and straightforward way to study how to perform the Lumbar Puncture procedure and will enable the student to train and perfect skills needed with almost no supervision required.

\section{Related Work}

There are many existing studies in the broader literature that have examined AR technology related to medical training. Learning in the medical domain is, to a large extent, workplace learning and involves mastery of complex skills that require performance up to professional standards in the work environment [10]. With Augmented and Virtual reality becoming cheaper, more reliable and more efficient, research applications are growing in the number of new possibilities for both medicinal practice and education. The aim of the study was to support practitioners in their everyday work and make the studying process more effective [10]. Traditional medical education involves studying the human body via literature, images, and models [10].

Over the past several decades, researchers have made small strides to provide medical professionals the most realistic work environment. These training tools can be physical devices, software applications, teaching methodologies, and more. Augmented Reality (AR) headsets and applications have steadily made advances to make it easier for teachers to assist students to learn. Studies show that there are a lot of gaps in AR research and have also identified what capacity the research needs assistance in [2] and some do not find any immediate advantage of an AR-enhanced training over traditional ways, nevertheless stating that AR-enhanced training gave better results in the long run [12]. In a previous study, surveys show that medical practitioners found these types of different approaches to be useful in diagnosing and tracking 
conditions which might lead to impairment or disability [3]. The study also highlights how AR can be used to train medical students to recognize such conditions or to undertake associated medical procedures [3].

While reviewing literature we discovered multitudes of AR application that are being studied for various medical procedures. The articles by [10], [9], [4], [14], [6], summarizing the usage of AR in the field of medicine and medical education, list numerous applications as replacing textbooks, flash-cards and charts by using AR to visualize human anatomy and organs in general, as well as special cases such as visualizing 3D lung dynamics and training laparoscopy skills and a system called DAQRI that provides an interactive human heart hologram, and helping with remote surgery by seeing the virtual interaction and receiving the live feedback or visualizing certain information about the patient body and organ states during the surgery. [10] finds that learning supported with AR technology enables ubiquitous, collaborative and situated learning, delivering a sense of presence, immediacy, and immersion that may be beneficial to the learning process. Reviewing over 2500 papers, [9] claims that AR apps are useful in medical care and help users to decrease the cost of practice and improve both the evaluation process and the training success rate and concludes AR applications having the potential to offer a highly realistic situated learning experience supportive of complex medical education.

During 2018 various applications for HoloLens AR headset have emerged, supporting both training and practice of medical professionals. [8] propose a complex system to assist clinical and nonclinical pathology. The system provided users with a virtual workplace, allowing them to view images of organs and related data, compare those images with organs in question and even perform video calls to narrow specialists and share data remotely for them to assist with questionable cases. [15] describe a system that enables an AR training environment for training in acetabular cup implant orientation, which traditionally is performed during actual surgeries, posing threat to the patient. [1] had researched possibilities of HoloLens AR headset to guide medical professionals in facet join injection procedures in order to accurately insert the needle. During the research, [1] manufactured a model representing the respective human body part, in which the needle was inserted by the headset's guidance, reporting a significant decrease in unsafe needle placement and time required to perform the operation in comparison to nonguided insertion. [5] utilized HoloLens to augment the manikin used for training in Basic Life Support and Defibrillation (BLSD) procedures. The implemented system was self-instructing and not only "adds" the missing body parts of the manikin, but also provides several virtual characters such as police officers and bystanders to make the training environment closer to real one. It must also be noted, that the manikin used in research by [5] was capable of providing feedback through special equipment without using HoloLens. In conjunction with research by [5], it is also interesting to mention research by [7]. Although [7] had not used a HoloLens device, they manufacture a manikin with haptic feedback designed to simulate the Lumbar Puncture procedure, with data derived from the feedback also used to model the current position of the human spine and needle in 3D and present to the student for better illustration of the student's actions. Positive results of both of these researche studies lay foundation for future integration of animated and haptic manikins into AR educational environment. Although all of the previous applications assisted in creating a template for the development of our system, it must be noted, that all the researche listed in the current paragraph, apart from research by [8], which was not a case of medical education, concentrate solely on the specific medical procedure in question, while we aim to leave the design abstract to enable relatively easy additions of new training environments in the future. 
AR to Enhance Training of Lumbar Puncture Procedure Sargsyan, Bassy, Xin, Sathish, Liu, Seals and White

\section{Requirements for the Future System}

We aim to create a suite of augmented reality training modules for the needs of medical education, specifically the training of the Lumbar Puncture procedure. The main objectives of the research are the following:

- Provide students with a library of educational materials and tutorials, that can be easily accessed via AR headset without interacting with any real physical objects.

- Augment the educational environment and existing real tools to establish a more realistic environment for student training.

- Perform testing of the student to ascertain their theoretical knowledge about Lumbar Puncture procedure.

- Supervise the student while performing the procedure, indicate the accuracy of the student's actions.

During the training process, the student will pass through three steps. For the first step, text and video materials will be shown to the student to familiarize them with the basics and key points of the Lumbar Puncture procedure. In the second step, the student will be able to inspect 360 degrees and interact with 3D models of the tools needed for operation, as well as the human body model. The student will be guided through the selection of appropriate tools and the steps of the Lumbar Puncture procedure. The student will also be required to show these actions on the 3D models, such as telling if the patient is positioned correctly and providing illustrations of where to insert the needle into the human body. In the third stage, the student will need to perform an AR guided operation on the actual manikin.

As already mentioned, a checklist of actions exists to perform a successful Lumbar Puncture procedure. For each entry in the checklist, the student is provided both text and video materials that he/she can go through before starting the corresponding action. Generalizing, we can write down the following functional requirements for the system:

1. Search and select the procedure

2. Observe the checklist and view the corresponding materials

3. Switch between text and video materials without navigating back to the checklist

4. Navigate to next and previous items in a checklist, without navigating back to checklist

5. Provide voice user interface (VUI) for hands-free interaction

6. Ability to remove all virtual elements from FOV and bring them back by request.

One of the advantages of the AR headset serving as a library for medical students and practitioners is that no interaction with physical objects (e.g. books, tablets) is required to retrieve the information, which is valuable in case of real medical operations. The VUI is also crucial, as it is typical to have both hands busy during medical procedures. Users of this system must also be able to free his/her sight from any virtual objects to get a clear image while performing medical operations.

The system must be able to add additional layers of interactivity to the training environment, enriching students' experience. When applied to the case of the Lumbar Puncture procedure, we can outline the following: 
AR to Enhance Training of Lumbar Puncture Procedure Sargsyan, Bassy, Xin, Sathish, Liu, Seals and White

- There are several contraindications for the Lumbar Puncture procedure. In the usual training setting, the student will be required to orally state the contraindications. With the AR headset, it will be possible to create a virtual environment, where the student must question the virtual patient for contraindications.

- The manikin for the Lumbar Puncture procedure training depicts only the lower part of the human body. To make the training more realistic, it must be possible to augment the manikin with missing body parts. Having the manikin extended to full body, we can test the student for the knowledge of the correct patient position required to safely perform the Lumbar Puncture procedure.

- It must also be possible to show the spine position in the manikin, and guidelines for the needle insertion. The student may choose between different levels of detail of the guidelines, slowly lowering it during the process of mastering the Lumbar Puncture procedure.

While these features are outlined with regard to the Lumbar Puncture procedure, medical training for various procedures require some or all of the mentioned features to be supported in addition to procedure specific features, such as correct patient position for the Lumbar Puncture procedure mentioned above.

\section{Development Process}

Understanding the requirements for a truly immersed environment we chose the Meta 2 headset by Metavision for our development platform. Compared with other devices like HoloLens, Meta has some advantages such as price and larger field of view (FOV) which provides a more complete AR experience, but requires to always be tethered to a computer.

The Meta 2 Headset has a 90 degree top, 80 degree bottom horizontal and a 50 degree vertical FOV, ending up with FOV much larger than one of HoloLens. Albeit researches by [15] [1], [5], [8] had not indicated any limitations due to the small FOV of HoloLens, we consider FOV to be an important component to create a realistic medical training environment. It not only provides more space to position virtual objects, but also gives the ability to realistically present virtual objects close to actual eye periphery, increasing chances to simulate different medical practice scenarios including ones were medical professional must be able to notice and respond to events going on near sight periphery, such as the patient trying to change position required by the procedure, or indicating pain or discomfort with slight body movements.

Unlike HoloLens and various other AR headsets, which can operate standalone, the Meta 2 headset must always stay connected to the computer. This is not a limitation for implementing the Lumbar Puncture procedure, but it can be for other medical procedures that might need more freedom of maneuver that a wire-connected device can provide. Nevertheless, we focus our research on outlining the features for training medical procedures, keeping the implementation abstract and portable, so this and possible other obstacles must not be a limitation for our system.

It was decided to implement the library feature of the system first. Metavision provides both $\mathrm{C}++$ and Unity SDKs for development. The Unity SDK was chosen, to support the possible transition to other headsets in the future. We have also utilized Google Cloud Speech-to-Text API to implement a voice user interface. The following Cassette Player Paradigm was used as the UI paradigm for the first version of the system.

The system must act as a library, providing text and video materials to the student. It was decided to give video materials higher priority, and build a Cassette Player Paradigm to 
create the system user interface. In this paradigm, each video tutorial was depicted as a $3 \mathrm{~d}$ VHS cassette, stored on a bookshelf. To view the tutorial, a student must grab it from the bookshelf and put it inside a $3 \mathrm{~d}$ virtual VHS player, which will "play" the "cassette" - showing the video on a virtual display. The bookshelf and VHS player can be moved in space, rotated and resized. Doing this, the student could position the VHS player in a suitable position, so he/she could perform the operation without being distracted by virtual objects, and easily view the materials when needed.

\section{Technical Issues}

In general, we find our experience with Metavision's Meta 2 headset highly satisfactory, with Metavision constantly improving the headset capabilities. Nonetheless, we want to discuss two main issues we have encountered during the development process, that also might be of interest to other AR headset developers, especially ones working with the Meta 2 headset.

\section{Peripheral sight and interaction with controls}

As already stated, one of the reasons that Meta 2 headset was chosen because it provided the highest FOV. During the design and development process, we attempted to make the most of this feature, but, while the user was able to adequately see displayed data, interaction with controls close to the peripheral sight zone was found to be difficult. Although Meta 2 headset was able to detect and process hand gestures on the periphery with acceptable precision, users found it to be hard for them to interact with those controls without irritation. It also appears that the way a user wears the headset slightly affects the user's and headset's capabilities of interacting on the periphery. These tend to decrease the real space available and the number of applicable UI layouts. These also uncover the issue that seeing hologram but being unable to adequately interact with it on the periphery may be a possible concern for Augmented Reality devices with wide FOV.

\section{Occlusion of the user interface}

Occlusion is still among the hottest issues in the field of Mixed Reality. Even though Metavision pays a lot of attention to the occlusion issue, the Meta 2 headset is still dependent on the environment and initial distance and position of a user and occluding object.

In the case of our system, it affected ability of the user to correctly overlay certain data on the manikin. The data was often not occluded correctly, resulting in it intersecting or becoming hidden inside manikin, causing frustration of the user. Currently, it was suggested to disable occlusion, until a solution is implemented to assist the headset in properly overlaying data.

Another current limitation of Meta 2 headset regarding occlusion is the lack of ability to disable or enable occlusion for specific virtual objects, the developer can disable or enable occlusion properties only for all virtual objects. We find that this technical issue is another one that can be addressed by AR headset makers.

\section{Results of Pilot Testing}

A pilot testing was conducted, involving 9 testers, 8 with computer science and 1 with a medical background, with the main focus on user interface and experience. Although testers had found the concept of the Cassette Player paradigm to be fun and engaging, as well as intuitive and 
easy to use, it was also noticed that the Cassette Player paradigm required excessive hand actions required to achieve the desired goal. This leads to an additional physical and cognitive load for the user and distracted from performing the Lumbar Puncture procedure.

\section{Conclusion}

Based on our experience and review of related work, we can state that AR headsets are capable of complementing and enhancing medical education processes and the medical field in general. While the reviewed researchers adopted Microsoft Hololens, we have used Metavision's Meta 2 headset. Albeit some issues covered in 5, our experience and pilot testing results have proven this headset to be suitable for medical education purposes, with its exceptional FOV promising interesting user experience with AR medical simulations. Preliminary testing had also exposed issues of the original Cassette Player paradigm and it was considered inefficient and not suitable for further development of the system. Based on this finding, it was decided to move to a more traditional user interface, optimizing it with regard to interaction limits between the user and an AR headset described in 5 .

\section{Future Work}

We find the current version of the software applicable for adaptation within the training process of the Lumbar Puncture operation. We look forward to more in-depth evaluation with a broader audience, including testing with actual medical students during actual Lumbar Puncture procedure studies. 3 .

We also plan to start development of the remaining features of the system, as described in

\section{References}

[1] Christoph A Agten, Cyrill Dennler, Andrea B Rosskopf, Laurenz Jaberg, Christian WA Pfirrmann, and Mazda Farshad. Augmented reality-guided lumbar facet joint injections. Investigative radiology, 2018.

[2] Murat Akçayır and Gökçe Akçayır. Advantages and challenges associated with augmented reality for education: A systematic review of the literature. Educational Research Review, 20:1-11, 2017.

[3] Ali Al-Khalifah, Rachel McCrindle, Paul Sharkey, and Vassil Alexandrov. Using virtual reality for medical diagnosis, training and education. International Journal on Disability and Human Development, 5(2):187-194, 2006.

[4] Mark Billinghurst, Adrian Clark, Gun Lee, et al. A survey of augmented reality. Foundations and Trends@ in Human-Computer Interaction, 8(2-3):73-272, 2015.

[5] Andrea Bottino, Pierluigi Ingrassia, Fabrizio Lamberti, Fernando Salvetti, Francesco Strada, and Antony Vitillo. Holo-blsd: an augmented reality self-directed learning and evaluation system for effective basic life support defibrillation training.

[6] Isabel CS da Silva, Gerson Klein, and Denise M Brandão. Segmented and detailed visualization of anatomical structures based on augmented reality for health education and knowledge discovery. Advances in Science, Technology and Engineering Systems Journal (ASTESJ), 2(3):469-478, 2017.

[7] Paul Gorman, Thomas Krummel, Roger Webster, Monica Smith, and David Hutchens. A prototype haptic lumbar puncture simulator. Studies in health technology and informatics, pages 106-109, 2000. 
AR to Enhance Training of Lumbar Puncture Procedure Sargsyan, Bassy, Xin, Sathish, Liu, Seals and White

[8] Matthew G Hanna, Ishtiaque Ahmed, Jeffrey Nine, Shyam Prajapati, and Liron Pantanowitz. Augmented reality technology using microsoft hololens in anatomic pathology. Archives of pathology \& laboratory medicine, 142(5):638-644, 2018.

[9] Jennifer Herron. Augmented reality in medical education and training. Journal of Electronic Resources in Medical Libraries, 13(2):51-55, 2016.

[10] Carolien Kamphuis, Esther Barsom, Marlies Schijven, and Noor Christoph. Augmented reality in medical education? Perspectives on medical education, 3(4):300-311, 2014.

[11] Kevin Kunkler. The role of medical simulation: an overview. The International Journal of Medical Robotics and Computer Assisted Surgery, 2(3):203-210, 2006.

[12] Christoph Noll, Ute von Jan, Ulrike Raap, and Urs-Vito Albrecht. Mobile augmented reality as a feature for self-oriented, blended learning in medicine: Randomized controlled trial. JMIR mHealth and uHealth, 5(9), 2017.

[13] Kathleen R Rosen. The history of medical simulation. Journal of critical care, 23(2):157-166, 2008.

[14] Iuliana Andreea Sicaru, Ciprian Gabriel Ciocianu, and Costin-Anton Boiangiu. A survey on augmented reality. Journal of Information Systems \& Operations Management, 11(2), 2017.

[15] Luke Western, K Logishetty, R Morgan, J Cobb, and E Auvinet. An enhanced microsoft hololens@ augmented reality platform for training in acetabular cup implant orientation: A randomized controlled trial. In Orthopaedic Proceedings, volume 100, pages 42-42. The British Editorial Society of Bone \& Joint Surgery, 2018. 